\title{
Erratum to: The Effects of Casting Porosity on the Tensile Behavior of Investment Cast 17-4PH Stainless Steel
}

\author{
D.F. Susan, T.B. Crenshaw, and J.S. Gearhart
}

\section{Erratum to: J. of Materi Eng and Perform (2015) 24:2917-2924 DOI 10.1007/s11665-015-1594-y}

Please note that all of the following text is missing from the PDF version of this article (though it is present in the HTML version of the article). This text should appear beginning at the very start of text on p. 2921 of the PDF:

\section{Effects of Casting Porosity on Ductility}

\section{Sample Geometry, Test Temperature, and Strain Rate}

The strain-to-failure results are summarized in Fig. 6 and 7 as a function of the measured porosity for H925 material. Recall that the area \% porosity was measured on the fracture surfaces, not in the bulk. Compared to the strength findings, porosity has a more pronounced effect on ductility. In Fig. 6(a), only $\sim 10 \%$ void area results in an almost $80 \%$ decrease in failure strain. The results are qualitatively similar to those found in the literature for other alloy systems (Ref 9-24). The quantitative fractography and metallography by Gokhale et al. illustrated the strong preference for fracture in regions of porosity in cast material (Ref 9-13). That is, the fracture process is not correlated to the average microstructure but, rather, it is related to the extremes in microstructure-local regions of high void content. In the present study, quantitative metallography on random cross-sections of several cast components indicated overall porosity was only $0.03 \%$ (.0003 volume fraction). This is a low value and well within specification limits for casting quality. The results here confirm that the fracture properties of cast $17-4 \mathrm{PH}$ cannot be predicted based on overall/average porosity. Note that the individually cast round bars were not included in the metallographic analyses; while the overall amount of porosity was still low, it is likely that higher $\%$ porosity would be obtained along the centerline of these samples.

In Fig. 6(a), the results obtained from separately cast round tensile specimens are denoted by the filled symbols. The data for small rectangular (machined from thin-wall components)

The online version of the original article can be found under doi: 10.1007/s11665-015-1594-y.

D.F. Susan, T.B. Crenshaw, and J.S. Gearhart, Sandia National Laboratories, Albuquerque, NM 87185, USA. Contact e-mail: dfsusan@sandia.gov. and large round tensile bars follow the same trend and the bestfit curve in Fig. 6(a) is obtained from the combined data. The two populations are combined in subsequent figures. This result is important since separately cast tensile bars are acceptable according to many specifications. Even so, it may be useful to test samples machined from actual components since the range in porosity may differ from cast test bars in some cases, especially for complex geometries and changes in wall thickness where restricted feeding of the solidification mushy zone may prevail.

Low temperature tensile tests, performed at $-10{ }^{\circ} \mathrm{C}$, are included in Fig. 6(b). At low temperature, the failure strain of "defect-free" material is only about $6 \%$, compared to about $9 \%$ at room temperature. The presence of internal casting voids lowers the ductility even further, with a similar but not identical trend compared to the room temperature results. Low ductility at cold temperatures is expected for cast $17-4 \mathrm{PH}$. With Charpy impact testing, Rack determined a ductile-to-brittle transition temperature in the vicinity of room temperature, even for the alloy in a low strength, overaged condition (Ref 2). Note that four samples in Fig. 6 were tested at strain rate of $1 \mathrm{~s}^{-1}$, two at room temperature and two at $-10{ }^{\circ} \mathrm{C}$. Within the experimental scatter, and with the limited number of tests, no significant trends were observed in the higher strain rate results.

\section{Quantitative Analysis Method 1: Index of Defect Susceptibility}

The effect of casting porosity on room temperature ductility for different heat treatments is shown in Fig. 7. In the low strength H1100 condition, the defect-free ductility is about 12$13 \%$. In comparison, approximately $14-16 \%$ strain to failure has been reported for cast $17-4 \mathrm{PH}$ in the overaged conditions $\mathrm{H} 1150 \mathrm{M}$ and H1200, presumably with no porosity (Ref 2, 5). As shown in Fig. 7, the presence of internal casting voids reduces the failure strain in $\mathrm{H} 1100$ material as well. For a given area fraction porosity on the fracture surface, the H1100 samples generally display higher strain to failure compared to the H925 condition. Failure strain data at room temperature for the H925 condition are reproduced from Fig. 6, but with different axis limits in Fig. 7. Only three samples were tested in the H1025 condition, and they all happened to be relatively defect-free, so it was not possible to develop a full trend for this heat treat condition. The failure strain of defect-free $\mathrm{H} 1025$ samples was in the range of $9-11 \%$, which does represent an intermediate ductility, as expected. The results in Fig. 7 show the extent to which precipitation heat treatment after casting can 
affect the alloy's ability to deform in the vicinity of internal flaws. Following the approach by Gokhale et al. (Ref 9-13), logarithmic best-fit curves are shown for the H925 and H1100 room temperature data sets.

No previous studies are available on the effects of heat treatment of cast $\mathrm{PH}$ stainless steel on sensitivity to casting flaws. For aluminum alloys, Lee tested the as-cast versus T6 tempered material and found that the T6 material was only slightly less sensitive to casting voids than the as-cast material. The author stated that the T6 versus as-cast results were remarkably similar given the observed differences in microstructure ( $\mathrm{Si}$ particle morphology) between the two conditions (Ref 22). In the present work, the as-cast condition was not tested since $17-4 \mathrm{PH}$ is typically not used in the as-cast condition.

By taking the logarithm of both sides of Eq 1, the index of defect susceptibility is given by the slope of the log-log plot as displayed in Fig. 8. The index of defect susceptibility ( $x$ value in Eq 1) is 15.1 for investment cast $17-4 \mathrm{PH}$ in the high-strength H925 condition. The lower strength H1100 condition gives an index of defect susceptibility of only $\sim 5.0$. In Table 1 , the results for $17-4 \mathrm{PH}$ are compared to the defect susceptibility parameters found in previous studies of cast aluminum and magnesium alloys. The comparisons indicate that $17-4 \mathrm{PH}$ has moderate defect susceptibility in the high-strength condition, comparable to some magnesium alloys, and that heat treatment to the H1100 condition reduces the index of defect susceptibility below any of the $\mathrm{Al}$ or $\mathrm{Mg}$ alloys.

Also note that on p. 2922 of the article PDF, the expression " $<$ Dummy RefID="Fig9" appears at the end of the first paragraph of text in the right-hand column when it should not. 